\title{
PHƯƠNG PHÁP XÂY DỰNG MIỀN ỔN ĐỊNH ROBUST
}

\author{
LÊ HÙNG LÂN
}

\begin{abstract}
The problem of construting a robust stability domain in paramater space is considered. The proposed technique is based on genelizating a classical $D$-partition method [1] for control system with uncertain parameters.
\end{abstract}

\section{I. ĐẶT VẤN ĐỀ}

Giả sử phương trình đặc trưng hệ thống tuyến tính:

$$
H(s, t, q,)=0
$$

phụ thuộc vào 2 nhóm tham số $t$ và $q$, trong đó $t$ là các tham số được chọn để xem xét, còn về $q$ ta chỉ biết là nó nằm trong hộp $Q$ nào đó. Hệ thống ổn định nếu nghiệm của phương trình (1) nằm bên trái mặt phẳng phức $s$.

Miền ổn định robust theo các tham số $t$ được coi là miền $Y(Q)$ trong không gian các tham số $t$, chứa các điểm mà ứng với nó tính ồn định hệ thống được đàm bảo với mọi giá trị $q \in Q$. Nếu $Q$ thu gọn thành một điểm thì miền ổn định robust trở thành miền ôn định thông thường.

Giới hạn ổn định theo các tham số $t$ với giá trị cố định $q \in Q$ được tạo nên từ các đường giới hạn $N_{\omega}$ hoặc các đường giới hạn kì dị $\bar{N}_{\omega}[2]$. Phương trình đường giới hạn miền ổn định này có thể mô tả qua dạng không tường minh sau:

$$
H(j \omega, t, q)=0 .
$$

Các đường giới hạn $N_{\omega}$ và $\bar{N}_{\omega}$ tạo nên miền ồn định $Y(Q)$. Khi có bất định tham số $q \in Q$ các đường giới hạn $N_{\omega}, \bar{N}_{\omega}$ trên được mở thành các băng thu hẹp miền ồn định $Y(q)$ thành $Y(Q)$.

Để xác định miền ổn định robust $Y(Q)$ có thể dựa trên việc xây dựng mỗi giá trị $\omega$ miền các giá trị $t$ cho tất cả $q \in Q[3]$. Miền các giá trị $t$ này sẽ được ký hiệu là $T(Q)$. Thực tế việc xác định $T(Q)$ không đơn giản. Độ phức tạp của nó phụ thuộc vào tính phi tuyến đa dạng của các tham số $t$ và $q$ có trong đa thức đặc 
trưng $H(s, t, q)$. Trong [3] có đề cập đến trường hợp đơn giản nhất khi phương trình đặc trưng có dạng

$$
H(s, q) t+R(s, q)=0,
$$

$R(s, q)$ - đa thức khoảng.

Trong [2], số hạng thứ hai được mở rộng cho dạng tuyến tính tồng quát hơn:

$$
R(s, q)=\sum a_{i} \phi_{i}(s)
$$

Trường hợp $t$ là các tham số bộ điều chỉnh PID được xét đến trong [4] cũng nằm trong nhóm có tính tuyến tính của số hạng đầu, mặc dù có xét đến tính phi tuyến bất kỳ của số hạng thứ hai.

Trong báo cáo này giả thiết trong trường hợp tổng quát từ phương trình (1) nhận được:

$$
t=f(\omega, q),
$$

$f: R^{m} \rightarrow R^{2}$ là phi tuyến bất kỳ.

Vấn đề đặt ra là xác định được miền giá trị $T(Q):=\{t, \forall q \in Q\}$. Điều này có thể đạt được trên cơ sở định lý sau.

\section{II. ĐINHH LÝ TÔNG QUÁT VỀ ẢNH CƯA PHÉP CHIẾU PHI TUYÊN}

Xét hàm liên tục đạo hàm được:

$$
f=Q \rightarrow R^{2},
$$

trong đó $Q$ là hộp của $R^{m}$

$$
Q=\left\{q=\left(q_{1}, \ldots, q_{m}\right): a_{i} \leq q_{i} \leq b_{i}, i=1, \ldots, m\right\}
$$

Ảnh hưởng của $Q$ được định nghĩa như $f(Q)$ :

$$
f(Q)=\left\{t \in R^{2}: t=f(q), q \in Q\right\}
$$

và giới hạn của nó là $\partial f(Q)$.

Ta nói rằng tập $K \subset Q$ là tập kiểm tra nếu ảnh của nó phủ $\partial f(Q)$ :

$$
f(K) \supset \partial f(Q)
$$


Ký hiệu $g_{i}$ là các đạo hàm riêng của $f$ :

$$
g_{i}(q)=\frac{\partial f(q)}{\partial q_{i}}, i=1, \ldots, m, q \in Q .
$$

Ta nói rằng điểm $q \in Q$ là điểm chính (principal point), nếu tồn tại $g \neq 0$, $g \in R^{2}$ sao cho

$$
\left(g_{i}, g\right)= \begin{cases}>0, & q_{i}=a_{i} \\ =0, & a_{i}<q_{i}<b_{i} \\ <0, & q_{i}=b_{i}\end{cases}
$$

Điều đó có nghĩa là với các biến "ự do" $\left(a_{i}<q_{i}<b_{i}\right)$ tất cả các đạo hàm riêng là song tuyến (và vuông góc với $g$ ), các đạo riêng $g_{i}$ với các biến "dưới" $\left(g_{i}=a_{i}\right)$ nằm trong nưa bên kia, hai nựa phân cách bằng đường trực giao với $g$.

Tập các điểm chính như trên được ký hiệu là $P$.

Định lý 1. Tập các điểm chính là tập kiểm tra:

$$
\partial f(Q) \subset f(P)
$$

Chứng minh. Giả sử $f(q) \in \partial(Q)$. Khi đó có thể rút ra các kết luận sau.

a. Tất cả các tọa độ tự do phải có các đạo hàm riêng song song với nhau. Giả thiết ngược lại, tức $a_{i}<q_{i}<b_{i}, a_{k}<q_{k}<b_{k}$ với $i, k$ nào đó và $g_{i}=\alpha g_{k}$, $\alpha \in R$. Khi đó với $\varepsilon_{1}, \varepsilon_{2}$ đủ nhỏ: $\left|\varepsilon_{i}\right|<\delta, \delta>0, i=1,2$ các điểm

$$
\begin{aligned}
q & =\left(q_{1}, \ldots, q_{m}\right), q_{i}=q_{i}+\varepsilon_{1} \\
q_{k} & =q_{k}+\varepsilon_{i}, q_{l}=q_{1} l \neq k, i
\end{aligned}
$$

thuộc vào $Q$.

Hàm $R^{2} \rightarrow R^{2}, \varphi(\varepsilon)=f(q), \varepsilon=\left(\varepsilon_{1}, \varepsilon_{2}\right)$ có đạo hàm riêng $\frac{\partial \varphi(0)}{\partial \varepsilon_{1}}=g_{i}(q)$, $\frac{\partial \varphi(0)}{\partial \varepsilon_{2}}=g_{k}(q)$, do đó Jacobian $J(\varepsilon)=\operatorname{det} \frac{\partial \varphi}{\partial \varepsilon}$ không bằng 0 tại 0 . Điều này có nghĩa là $\varphi(0)$ là điểm trong của $\varphi\left(Q_{\delta}\right), Q_{\delta}=\left\{\varepsilon \in R^{2}:\left|\varepsilon_{1}\right|<\delta,\left|\varepsilon_{2}\right|<\delta\right\}$.

Do đó $f(q)$ là điểm trong của $f(Q)$ và $f(q) \notin \partial f(Q)$.

b. Tất cả các đạo hàm riêng khác không ứng với các tọa độ "trên", "dưới" phải được phân tách ra bởi các đạo hàm riêng ứng với biến tự do, tức nếu $g_{i}(q) \neq 0, a_{i}<q_{i}<b_{i}$, thì dấu của tích $\left(g_{k}(q), g_{i}(q) \cdot e^{j 90}\right)$ là giống nhau với mọi $k$ có $q_{k}=a_{k}$, và ngược lại với mọi $k: q_{k}=b_{k}$. Giả thiết điều đó là không đúng, tức $\left(g_{k}(q), g_{i}(q) \cdot e^{j 90}\right)>0, q_{k}=a_{k}$ và $\left(g_{l}(q), g_{i}(q) \cdot e^{j 90}\right)>0, q_{l}=b_{l}$ với $i$ nào đó: $a_{i}<q_{i}<b_{i}$. Xét diểm:

$$
q=\left(q_{1}, \ldots, q_{m}\right), q_{i}=q_{i}+\varepsilon_{1}, q_{k}=q_{k}+\varepsilon_{2}, q_{l}=q_{l}-\varepsilon_{3} .
$$


Khi đó tồn tại $\delta>0$ sao cho:

$q \in Q$ khi $\varepsilon \in Q_{\delta}, Q_{\delta}=\left\{\varepsilon=\left(\varepsilon_{1}, \varepsilon_{2}, \varepsilon_{3}\right):\left|\varepsilon_{1}\right| \leq \delta, 0 \leq \varepsilon_{2} \leq \delta, 0 \leq \varepsilon_{3} \leq \delta\right\}$

Hàm 3 biến $\varphi: R^{3} \rightarrow R^{2}, \varphi(\varepsilon)=f(q)$ có các đạo hàm riêng tại 0 :

$$
\frac{\partial \varphi(0)}{\partial \varepsilon_{1}}=g_{i}(q), \frac{\partial \varphi(0)}{\partial \varepsilon_{2}}=g_{k}(q), \frac{\partial \varphi(0)}{\partial \varepsilon_{3}}=-g_{l}(q)
$$

và

$$
\varphi(\varepsilon)=\underbrace{\varphi(0)+\varepsilon_{1} g_{i}(g)+\varepsilon_{2} g_{k}(q)-\varepsilon_{3} g_{l}(q)}_{\psi(\varepsilon)}+\sigma(\varepsilon) .
$$

Theo giả thiết các vectơ $g_{k}(q)$ và $-g_{l}(q)$ nằm ở hai nửa mặt phằng khác nhau (do $g_{k}(q)$ và $g_{l}(q)$ ở cùng trong một nứa mặt phẳng), phân chia bởi vectơ $g_{i}(q)$. Vî vậy $0 \notin \partial \psi\left(Q_{\delta}\right)$ (vì hình nón tạo ra bởi 4 vectơ $g_{i}(q),-g_{i}(q), g_{k}(q),-g_{l}(q)$ phư toàn bộ mặt phằng). Điều này cũng đúng cho hàm $\varphi(\varepsilon)$. Nói cách khác $f(q)$ không phải là điểm giới hạn của $f(Q)$.

c. Nểu không có tọa độ tự do nào, các đạo hàm riêng ứng với các tọa độ trên, dưới phải nằm ở các nửa mặt phẳng khác nhau. Điều này có thể được chứng minh tương tự như chứng minh trên.

Kết hợp các khằng định trên ta được điều phải chứng minh.

Với định lý này thủ tục tìm các điểm chính $P$ tạo nên miền giá trị $T(Q) \cong$ $f(Q)$ như sau:

- Tìm các đạo hàm riêng $g_{i}=\partial f / \partial q_{i}, i=1, \ldots, m, g_{i} \in R^{2}$.

- Tìm trong số các $g_{i}$ trên nhóm gồm $k$ phần tử, $k=2, \ldots, m$, là các vectơ song tuyến với nhau:

$$
g_{j} \| g_{l}, j \neq l \text {. }
$$

Các giá trị $q_{i}$ tìm được là một trong hai nhóm giá trị tọa độ để xác định điểm chính.

- Nhóm giá trị thứ hai là các tọa độ đỉnh $a_{i}$ hoặc $b_{i}$ còn lại (có tính lần lượt các đ̛̉inh trong khi các biến còn lại thay đổi).

- Kết hợp các nhóm giá trị tham số tìm được trên ta được tập các điểm chính $P$ cần xác định.

Thực tể có thể nhận được nihững các kết quả đơn giản hơn trong số trường hợp đặc biệt, thường gặp của hàm $f$. 


\section{Các hàm có tính chất $D$}

- Giả sứ với mọi $q \in Q$ và mọi $k=1, \ldots, m, i \neq k$ có:

$$
g_{k}(q) \neq 0, g_{i}(q) \neq \alpha g_{k}(q), \alpha \in R \text {. }
$$

Những hàm có các hướng đạo hàm riêng khác nhau như vậy được gọi là hàm có tính chất $\mathrm{D}$ (distinct directions of derivatives).

Giả sử chúng được sắp xếp theo thứ tự tăng dần của argument

$$
\arg g_{1}<\arg g_{2}<\cdots<\arg g_{m} .
$$

Định nghĩa $2 m$ cạnh của $Q$ sau là các cạnh chính (principal edge) $(\mathrm{PE})$ :

$$
\begin{gathered}
E_{i}^{+}=\left\{q: q_{k}=a_{k}, k<i ; a_{i} \leq q_{i} \leq b_{i}, q_{k}=b_{k}, k>i\right\} \\
E_{i}^{-}=\left\{q: q_{k}=b_{k}, k<i ; a_{i} \leq q_{i} \leq b_{i}, q_{k}=a_{k}, k>i\right\} \\
P E=\bigcup_{i=1}^{m}\left(E_{i}^{+} \cup E_{i}^{-}\right) .
\end{gathered}
$$

Hệ quả 1. Các cạnh chính là tập kiểm tra cho các hàm có tính chất D:

$$
\partial f(Q) \subset f(P E)
$$

Ví dụ: Giả sử $m=3, q_{i} \leq a_{i} \leq b_{i}, i=1,2,3, \arg g_{1}<\arg g_{2}<\arg g_{3}$. Khi đó các cạnh chính của hộp $Q$ như sau:

$$
\begin{aligned}
& E_{1}^{+}=\left\{\underline{q_{1}} \leq q_{1} \leq \overline{q_{1}} ; q_{2}=\overline{q_{2}} ; q_{3}=\overline{q_{3}}\right\} \\
& E_{1}^{-}=\left\{\underline{q_{1}} \leq q_{1} \leq \overline{q_{1}} ; q_{2}=\underline{q_{2}} ; q_{3}=\underline{q_{3}}\right\} \\
& E_{2}^{+}=\left\{q_{1}=\underline{q_{1}} ; \underline{q_{2}} \leq q_{2} \leq \overline{q_{2}} ; q_{3}=\overline{q_{3}}\right\} \\
& E_{2}^{-}=\left\{q_{1}=\overline{q_{1}} ; \underline{q_{2}} \leq q_{2} \leq \overline{q_{2}} ; q_{3}=\underline{q_{3}}\right\} \\
& E_{3}^{+}=\left\{q_{1}=\underline{q_{1}} ; q_{2}=\underline{q_{2}} ; \underline{q}_{3} \leq q_{3} \leq \overline{q_{3}}\right\} \\
& E_{3}^{-}=\left\{q_{1}=\overline{q_{1}} ; q_{2}=\overline{q_{2}} ; \underline{q}_{3} \leq q_{3} \leq \overline{q_{3}}\right\}
\end{aligned}
$$

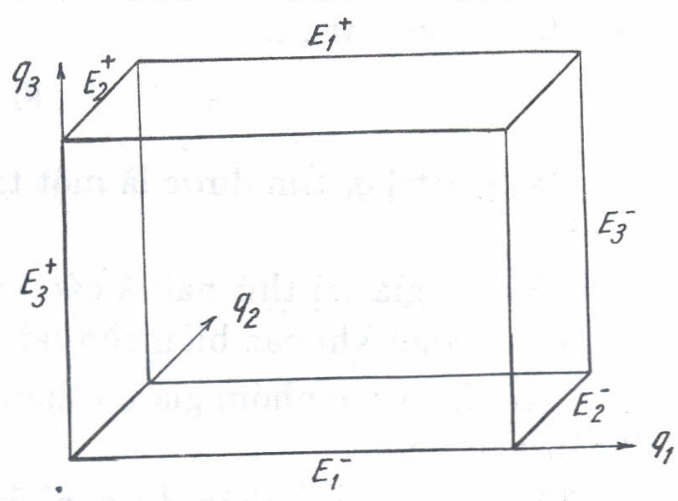

Hinh 1 


\section{Các hàm hữu tî với cấu trúc phân lớp}

Xét hàm:

$$
f(q)=\frac{N_{0}+\sum_{i=1}^{n} N_{i}\left(q^{i}\right)}{D_{0}+\sum_{i=1}^{n} D_{i}\left(q^{i}\right)},
$$

$$
\begin{aligned}
& q=\left(q^{1}, \ldots, q^{n}\right) \in R^{m}, q^{i} \in R^{m_{i}}, \\
& q^{i} \in B^{i}=\left\{q^{i}: a_{k}^{i} \leq q_{k}^{i} \leq b_{k}^{i}, k=1, \ldots, m_{i}\right\}, \\
& B=B^{1} \times \cdots \times B^{n},
\end{aligned}
$$

trong đó $N_{i}: R^{m_{i}} \rightarrow C, D_{i}: R^{m_{i}} \rightarrow C$ là các hàm đa tuyến (tuyến tính với từng tham số nếu các tham số khác cố định).

Ta nói rằng $q=\left(q^{1}, \ldots, q^{n}\right) \in B$ nằm trong cạnh mở rộng của $B$ nếu nhiều nhất có một $q^{i}$ không phải là đỉnh cúa $B^{i}$ :

$$
q^{i} \in B^{i}, q^{k} \in V^{k}, k \neq i
$$

$\left(V^{k}\right.$ là tập hợp các đỉnh cúa $\left.B^{k}\right)$.

Hệ quả 2. Cách cạnh mở rộng của B là tập kiểm tra cho hàm (14).

\section{Các hàm hữu tỷ với các biến độc lập}

Nếu các biến $̛$ tư sớ và mẫu số của hàm (14) là độc lập với nhau thì hệ quả 2 có thể được phát biểu cụ thể hơn.

Giả sữ:

$$
\begin{gathered}
f(q)=\frac{N_{0}+\sum_{i=1}^{t} f_{i}\left(q^{i}\right)}{D_{0}+\sum_{i=t+1}^{n} f_{i}\left(q^{i}\right)}, q=\left(q^{1}, \ldots, q^{n}\right) \in R^{m}, q^{i} \in R^{m_{i}}, \\
\sum_{i=1}^{n} m_{i}=m, q^{i} \in B^{i}, B=B^{1} \times \cdots \times B^{n},
\end{gathered}
$$

trong đó: $f_{i}: R^{m_{i}} \rightarrow C$ là các đa tuyến và $D_{0}+\sum_{i=t+1}^{n} f_{i}(q) \neq 0$ với $q^{i} \in B^{i}$.

Ta nói rằng $q=\left(q^{1}, \ldots, q^{n}\right) \in B$ nằm trong cạnh chính mớ rộng cúa $B$ nếu với $1 \leq i \leq n$ nào đó $q^{i}$ là điểm chính của $f_{i}$ trên $B^{i}$ và các $q^{k}$ khác là các đỉnh chính của $f_{k}$ trên $B^{k}$ :

$$
q^{i} \in P_{i}, q^{k} \in P V_{k}, k \neq i
$$


( $P$ - tập các điểm chính, $P V$ - tập các đỉnh đồng thời là điểm chính).

Hệ quả 3. Các cạnh chính mở rộng của $B$ là tập kiểm tra cho hàm (15).

\section{MIỀN ỔN ĐỊNH ROBUST MẠCH NỐI TIẾP}

Mặc dù có thể coi định lý trên là phương pháp tổng để miền ổn định robust, thực tế việc tính toán cũng không đơn giản, chủ yếu do tính phức tạp của hàm $f(\dot{\omega}, q)$ trong $(5)$. Tuy nhiên nếu trong cấu trúc mạch hởi hệ thống có thể phân thành hai phần nối tiếp nhau, một phần chứa các tham số $t$ cần quan tâm, phần còn lại chứa các tham số bất định $q$ (hình 2$)$ :

$Z(\cdot),(q(\cdot)$ - các hàm phi tuyến, thì việc tính toán được đơn giản đi nhiều nhờ định lý 2 sau [5].

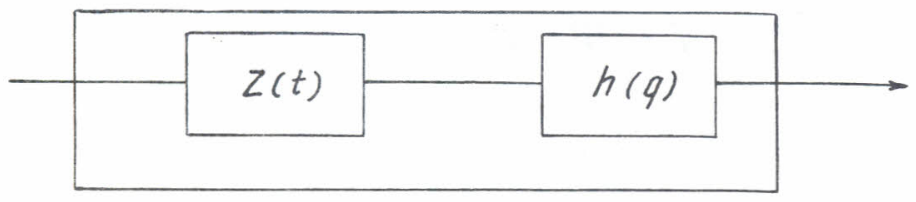

Hình 2. Mạch hở hệ thống

Định lý 2. Tập kiểm tra của hàm $h(\omega, q)$ cũng chính là tập kiểm tra của hàm $t=(\omega, q)$.

Nếu chú ý ta sẽ thấy thực tế cấu trúc hệ thống như trên rất hay gặp, chăng hạn khi các tham số $t$ nằm trong bộ điều chình, còn các tham số bất định có chứa trong mô hình đối tượng (hình 3 ):

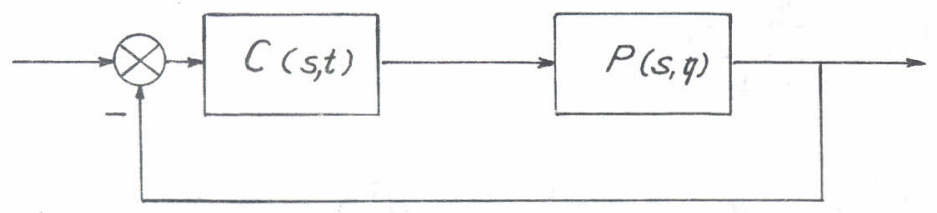

Hinh 3

Cụ thể lúc này có thể xáy ra 3 trường hợp:

$+t=\left(t_{1}, t_{2}\right)$ nằm ơ tử số hàm truyền bộ điều chỉnh (chằng hạn trường hợp PID đã được đề cập trong [4]). Phương trình đặc trưng có dạng:

$$
t_{1} A_{1}(j \omega)+t_{2} A_{2}(j \omega)=-A_{3}(j \omega) P^{-1}(j \omega, q)+A_{4}(j \omega),
$$


$A_{i}(j \omega), i=1, \ldots, 4$ - các hằng số phức.

$+t=\left(t_{1}, t_{2}\right)$ nằm ờ mẫu số. Khi đó:

$$
t_{1} B_{1}(j \omega)+t_{2} B_{2}(j \omega)=-B_{3}(j \omega) P(j \omega, q)+B_{4}(j \omega),
$$

$B_{i}(j \omega), i=1, \ldots, 4$ - các hằng số phức.

$+t_{1}$ ơ tử số, $t_{2}$ ơ mẫu số (hoặc ngược lại). Lúc này:

$$
\frac{t_{1} A_{1}(j \omega)+A_{2}(j \omega)}{t_{2} B_{1}(j \omega)+B_{2}(j \omega)}=-P(j \omega, q)
$$

Từ các dạng phương trình đặc trưng trên có thể rút ra hàm $t=f(\omega, q)$. Nội dung định lý 2 thể hiện ở chỗ các điểm chính $q^{*} \in Q$ tạo nên giới hạn miền $\partial P(j \omega, q)$ cũng chính là các điểm tạo nên giới hạn miền $\partial f(j \omega, q)$. Hàm truyền $P(j \omega, q)$ nói chung đơn giản hơn $f(j \omega, q)$ và quen thuộc hơn - đó là miền giá trị mô hình bất định. Công cụ tìm các điểm chính $q^{*}$ một cách tổng quát là định lý tương tự như định lý 1 cho phép chiếu phi tuyến $f: R^{n} \rightarrow C$.

\section{KẾT LUẬN}

Trong các bài toán về điều khiền robust một trong những vấn đề khó khăn nhất, chưa được đề cập nhiều là tìm ra các bộ điều chỉnh robust cho một đối tượng bất định cho trước. Phương pháp phân chia $D$ là một phương pháp tổng quát cho phép giải quyết bài toán đặt ra.

Ngoài ra khái niệm ổn định robust ở đây có thể được mở rộng hơn khi đưa ra các khái niệm về ổn định tuyệt đối, tương đối với các độ dự trữ $d, \alpha$ cần thiết [4]. Cảc kết luận trong bài khi đó vẫn giữ nguyên giá trị.
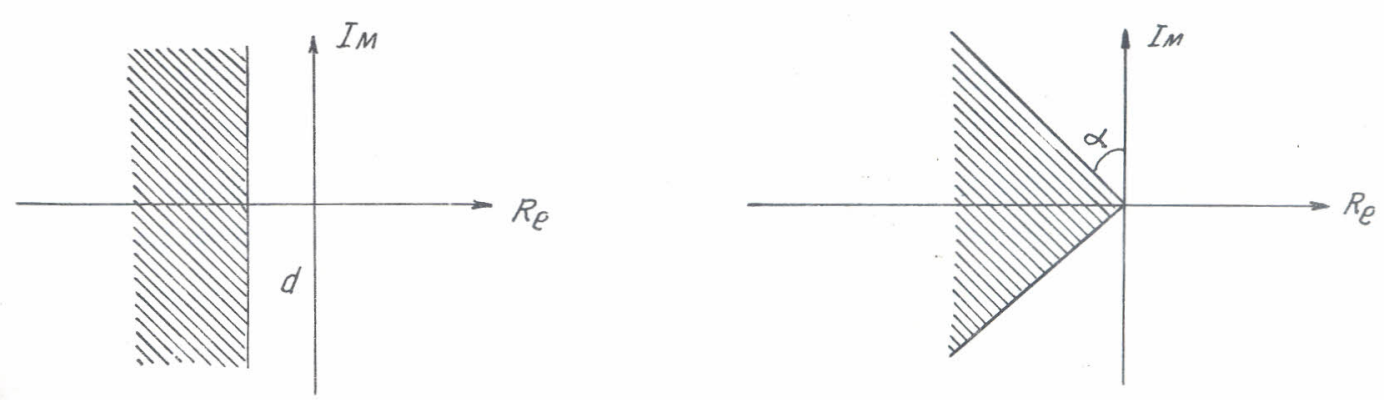

TÀI LIỆU THAM KHẢO

1. Neimark Yu. I., Ustoichivost linearizovanukh sistem. Leningrad, 1949. 
2. Neimark Yu. I., Oblast robastnoi ustoichivosti i robustnost po nelineimum parametram. Dok. Akademii Nauk, T. 235. N. 3 (1992) 438-440.

3. Petrov N.P., Polyak B.T., Robastnoe D razbienie. Avtomatika i Telemekhanika, 11 (1991) 41-53.

4. Lê Hùng Lân, Tông hợp bộ điều chỉnh PID robust thông qua phuơng pháp phân rã $D$. Tạp chí Tin học và Đî̀u khiển, T. 12, số 1 (1996).

5. Lê Hùng Lân, Phương pháp xây dụng phân rã $D$ robust trong không gian tham số bộ điều chinhh. Thông tin KHKT trưòng Đại học GTVT 1996, số 1, Tr. 58-53.

Truòng Đại học Giao thông Vận tải

Nhận bài ngày 2-6-1996 